\section{Inactividad física en Chile: debemos responder al llamado global}

\section{Physical inactivity in Chile: we must answer the global call}

\section{Señor Editor:}

Los beneficios de la actividad física a nivel individual y poblacional han sido bien documentados, no solo en términos de salud, sino que también en términos de sostenibilidad. Lamentablemente en Chile, solo 3 de cada 10 niños menores de 5 años cumple con las recomendaciones internacionales de actividad física ( $3 \mathrm{~h}$ diarias de actividad física moderada a vigorosa), mientras que en los adolescentes solamente 2 de cada 10 las cumple ( $1 \mathrm{~h}$ diaria de actividad física moderada a vigorosa $)^{1}$. En población adulta, los datos actuales no son más alentadores, ya que solamente un 15\% de los adultos reporta realizar actividad física en el tiempo libre según la Encuesta Nacional de Salud 2009-2010².

Recientemente la Organización Mundial de la Salud (OMS) ha lanzado una estrategia para desarrollar un nuevo Plan de Acción Global para Promover Actividad Física $^{3}$. Este nuevo Plan será presentado en mayo de 2018 en la $71^{\text {va }}$ Asamblea Mundial de Salud de la OMS. Este plan se desarrollará por medio de un trabajo colaborativo entre organismos internacionales, gobiernos, academia y sociedad civil. El objetivo de este plan será generar una visión, objetivos estratégicos y acciones $\mathrm{o}$ intervenciones para todos los actores y grupos de interés, que, al ser implementadas colectivamente, disminuyan los niveles de inactividad física globalmente.

Chile tiene un paso adelantado debido a que recientemente se lanzó la "Nueva Política de Actividad Física y Deporte" (http://www.mindep.cl/quienes-somos/ politica-nacional), sin embargo, el plan y métodos de implementación requerirán un trabajo exhaustivo de seguimiento para garantizar buenos resultados. El plan de acción global debería potenciar la política nacional, fomentando esfuerzos coordinados en distintos niveles y ministerios, y así respaldar el involucramiento de recursos suficientes para su implementación.

La Sociedad Internacional de Actividad Física y Salud (ISPAH) lideró la "Declaración de Bangkok en Actividad Física para la Salud Global y Desarrollo Sostenible" (BKKD) que fue lanzada en Noviembre de $2016^{4}$. La BKKD identificó oportunidades de priorización e implementación urgente para apoyar y promover la reducción de la inactividad física y contribuir a alcanzar 8 objetivos específicos de la Agenda Internacional de Desarrollo Sostenible (SDGs) de la Organización de Naciones Unidas (ONU) (Tabla 1). La BKKD es un gran avance para la búsqueda de objetivos transversales para distintos estamentos gubernamentales a través de la promoción de actividad física. Por ejemplo, la promoción del transporte activo (caminata o ciclismo) tiene reconocidos efectos positivos en salud pública, pero también contribuye a la construcción de ciudades y comunidades conectadas física y socialmente, a empoderar a ciudadanos para reclamar por mejores espacios públicos, promover la reducción de contaminación ambiental, y mejoras en accesibilidad y movilidad ${ }^{5}$. Por lo tanto, la BKKD es un llamado a los profesionales de salud, investigadores y tomadores de decisión para valorar integralmente estrategias de promoción de salud no solo en los individuos sino también en la comunidad en que están inmersos.

Las iniciativas globales antes mencionadas han ido también de la mano de alianzas internacionales del mundo académico y sociedades científicas. Dentro de estas alianzas está el Observatorio Global de Actividad Física (Global Observatory for Physical Activity, GOPA) que ha coordinado la participación de al menos 139 países de todos los continentes para elaborar un reporte de cada país en indicadores asociados a la actividad física (www.globalphysicalactivityobservatory.com) ${ }^{6}$. Los indicadores de GOPA ayudan a informar el estado de avance global en vigilancia epidemiológica, implementación de políticas y la capacidad de investigación en el área. Otra iniciativa es la Alianza global por niños activos y saludables (Active Healthy Kids Global Alliance, AHKGA) que en sus objetivos incluye la elaboración de un reporte global de actividad física(AF) infantil (www.activehealthykids.org). Cada país participante elabora un reporte de indicadores asociados a la $\mathrm{AF}$ a nivel individual, ambiental y político. En la última versión, Chile estuvo entre los países con las calificaciones más bajas a nivel global, con marcadas inequidades de género y socioeconómicas, evidentes en gran parte de los indicadores (www.chileactivate.cl) $)^{1}$. Las iniciativas de vigilancia mencionadas tienen el potencial de convertirse en importantes instrumentos de influencia para el desarrollo de planes, políticas y apoyo para la priorización en investigación para cada país.

\section{¿Cómo podríamos actuar desde Chile en pro de una población más activa?}

Para ayudar a revertir el negativo panorama en Chile es urgente fortalecer distintos ejes que permitan responder a este llamado internacional. Se requiere generar alianzas y compromisos tanto de organizaciones gubernamentales como no gubernamentales, academia, sociedades científicas y profesionales de salud, dispuestos a liderar este proceso de cambio.

Las organizaciones académicas y profesionales deben vincularse con su comunidad para establecer alianzas que respondan a las necesidades de su entorno a través de procesos participativos para el diseño de planes regionales o comunales de actividad física. Debemos fortalecer la formación de profesionales actualizados e integrales que respalden el abordaje de esta crisis, con especial énfasis en la promoción o fomento de salud en nuestros contextos. Debemos mejorar el intercambio de experiencias entre instituciones y organizaciones a nivel nacional e internacional para diseñar y adaptar interven- 
Tabla 1. Objetivos de desarrollo sostenible incluidos en la BKKD y las acciones que podrían contribuir en la disminución de la inactividad física

\begin{tabular}{|c|c|}
\hline $\begin{array}{l}\text { Objetivos de desarrollo sostenible } \\
\text { incluidos en BKKD }\end{array}$ & $\begin{array}{l}\text { Acciones para contribuir a la disminución de la inactividad } \\
\text { física }\end{array}$ \\
\hline SDG 3. Salud y bienestar & $\begin{array}{l}\text { 1. Renovar compromisos para invertir e implementar acciones políticas por } \\
\text { la actividad física en el curso de vida. }\end{array}$ \\
\hline SDG 4. Educación de calidad & $\begin{array}{l}\text { 2. Establecer plataformas de participación y coordinación nacional multi- } \\
\text { sectorial }\end{array}$ \\
\hline SDG 5. Igualdad de género & 3. Desarrollar capacidades de profesionales \\
\hline SDG 10. Reducción de desigualdades & 4. Aumentar la asistencia técnica y compartir experiencias \\
\hline SDG 11. Ciudades y comunidades sostenibles & 5. Fortalecer monitoreo y vigilancia \\
\hline SDG 13. Acción por el clima & 6. Apoyar y promover la colaboración, investigación y evaluación de políticas \\
\hline \multicolumn{2}{|l|}{ SDG 15. Vida de ecosistemas terrestres } \\
\hline SDG 16. Paz, justicia y sociedades inclusivas & \\
\hline
\end{tabular}

Abreviación: BKKD: Declaración de Bangkok en Actividad Física para la Salud Global y Desarrollo Sostenible.

ciones efectivas para nuestra realidad. El llamado a los profesionales de la salud pública es a diseñar programas integrales de promoción de actividad física para una modificación de conductas, integrando recursos como el urbanismo y el transporte, pertinentes a la realidad en que residen las personas, para individuos de diferentes edades, intereses, capacidades y posibilidades. En este punto la atención primaria en Chile puede ser un líder a nivel mundial debido a su estructura de asistencia y alta cobertura. Desde la investigación se debe contribuir a la evaluación de políticas y vigilancia epidemiológica, incluyendo también a grupos no representados actualmente, como la población infantil, adultos mayores y personas en situación de discapacidad. Además, debe tenerse en cuenta un modelo de descentralización nacional y regional (incluir pueblos y ciudades no capitales, por ejemplo) que incremente la pertinencia de las estrategias. Todo lo anterior debe estar conectado a un modelo de determinantes sociales de salud, especialmente aplicado a nuestro contexto de marcada desigualdad socioeconómica y de género observada en los diversos indicadores ligados a la inactividad física ${ }^{1}$.

Chile no debe seguir esperando. El llamado a la acción es urgente, pero revertir esta situación depende del accionar conjunto y organizado de varios sectores. El llamado global en desarrollo busca dar un nuevo impulso para que asumamos de modo más responsable y estratégico la responsabilidad de cambiar nuestra sociedad, con conciencia sobre las necesidades y características específicas de nuestro territorio y población.

\footnotetext{
Nicolas Aguilar-Farías ${ }^{1,2}$, Pía Martino-Fuentealba ${ }^{1,2}$, Gonzalo Infante-Grandon ${ }^{1,2}$, Andrea Cortinez-O'Ryan ${ }^{1,2,3}$
} ${ }^{1}$ Departamento de Educación Física, Deportes y Recreación. Universidad de La Frontera, Chile. ${ }^{2}$ Grupo de Investigación UFRO Activate,
Universidad de La Frontera, Chile. ${ }^{3}$ Departamento de Salud Pública, Pontificia Universidad Católica de Chile.

\section{Referencias}

1. Aguilar-Farías N, Cortinez-O'Ryan A, Sadarangani KP, Von Oetinger A, Leppe J, Valladares M, et al. Results From Chile's 2016 Report Card on Physical Activity for Children and Youth. Journal of physical activity \& health 2016; 13 (11 Suppl 2): S117-S23. doi: 10.1123/jpah. 2016-0314. PubMed PMID: 27848748 .

2. Ministerio de Salud de Chile. Encuesta Nacional de Salud ENS Chile 2009-2010. 2011.

3. World Health Organization. Governance: Development of a draft global action plan to promote physical activity 2017 . Available from: http://www.who.int/ncds/governance/physical_activity_plan/en/.

4. International Society for Physical Activity Health (ISPAH). The Bangkok Declaration on Physical Activity for Global Health and Sustainable Development. Br J Sports Med. 2017;51(19):1389-91. Epub 2017/06/24. doi: 10.1136/bjsports-2017-098063. PubMed PMID: 28642224.

5. Hipp JA, Bird A, van Bakergem M, Yarnall E. Moving targets: Promoting physical activity in public spaces via open streets in the US. Preventive medicine. 2017;103S:S15-S20. Epub 2016/10/30. doi: 10.1016/j.ypmed.2016.10.014. PubMed PMID: 27773707.

6. Hallal PC, Martins RC, Ramirez A. The Lancet Physical Activity Observatory: promoting physical activity worldwide. Lancet. 2014;384(9942):471-2. doi: 10.1016/S01406736(14)61321-0. PubMed PMID: 25110267. 